\title{
EXAMEN POLLINIQUE DE QUELQUES MIELS UNIFLORAUX
}

\author{
PAR
}

\author{
E. BARBIER
}

Laboratoire apicole, Nice.

I, 'analyse pollinique des miels, telle qu'elle a été utilisée à ses débuts, avait surtout pour but de différencier les miels exotiques des miels européens produits en Allemagne et en Suisse, par la présence de pollens caractéristiques des plantes d'outre-mer.

Par ailleurs l'examen des miels européens permettait de dresser leur composition pollinique et de constater des différences sensibles entre les divers miels. I)ans les conditions normales de l'apiculture on considérait surtout les proportions des différents pollens et on admettait qu'un miel était récolté sur une plante apicole donnée quand la proportion de pollen de cette plante daus le spectre pollinique atteignait ou dépassait $45 \mathrm{p}$. roo.

'Toutefois Maurizio notait qu'il existait des exceptions pour certaines plantes à pollen particulièrement abondant: Myosotis, Castanea, et signalait des miels extrêmement pauvres en pollens, cas de certains miels de labiées ou de Robinia.

En Irance, depuis une vingtaine d'années, sous l'influence de conditions économiques favorables, l'apiculture subit une évolution rapide et profonde.

A l'apiculture d'avant I940 héritière directe de l'apiculture ancestrale caractérisée par des ruches fixes, se substitue une apiculture spéculative qui donne naissance à des produits nouveaux : pollen et gelée royale. Par ailleurs les méthodes modernes d'exploitation des ruches basées sur le nourrissement stimulant et la transhumance font apparaître, grâce à la diversité de la flore française, des miels particuliers correspondant souvent à la floraison d'une seule espèce botanique. Certains de ces miels étaient tout à fait insoupçonnés, tels les miels de Lippia, Paliurus à côté de miels rares : Satureia, Lavandula, Rosmarinus.

Depuis quelques années je me suis attaché à l'étude de ces niels unifloriaux et à leur caractérisation ; leur couleur est souvent très éloignée 
des tons moyens des miels " toutes fleurs" et par ailleurs ils présentent entre eux de grandes variations de richesse et de composition pollinique.

Ces variations sont dues à plusieurs facteurs dont les plus importants apparaissent liés à la conformation des fleurs, à l'abondance de la miellée et des pollens et peut-être aussi à l'abeille elle-même qui n'extrait pas forcément avec autant de facilité ou d'avidité, du nectar contenu dans son jabot, les pollens des différentes plantes.

\section{Choix des éehantillons.}

Parmi les nombreux échantillons de miels adressés par les apiculteurs ne sont retenus dans cette étude, que ceux ayant une origine certaine, provenant d'apiculteurs faisant la sélection des miels à l'occasion de la transhumance ou après une floraison bien déterminée et qui fournissent des renseignements précis sur le mode d'extraction ; sont également retenus les échantillons présentés en sections bien homogènes.

Ces échantillons dans leur ensemble sont des miels commerciaux obtenus par extraction centrifuge et correspondent à des miels unifloraux, tels que les apiculteurs peuvent les récolter.

\section{Examen des pollens.}

L'examen pollinique de ces échantillons est effectué de la façon suivante

L.e sédiment provenant de la centrifugation de 20 grammes de miel est remis en suspension dans une quantité d'eau, telle que le mélange eaupollen atteigne le volume de $0, \mathrm{I} \mathrm{cm}^{3}$.

Le dénombrement des pollens se fait sur I/Io de ce volume, par prélèvement avec une pipette jaugée et après montage à la gélatine glycérinée colorée. Pour certains miels très pauvres en pollen le dénombrement est fait sur une quantité plus grande, mais dans tous les cas les résultats sont calculés pour ro g de miel.

Le spectre pollinique est établi sur roo grains après détermination des différents pollens rencontrés dans cette première préparation, ou dans le montage réalisé avec tout le reste du culot de centrifugation qui est préparé sur la même plaque.

\section{Résultats obtenus.}

Si on considère ces miels ayant une origine florale certaine on peut constater de grandes différences dans les résultats fournis par l'analyse pollinique, et qui sont rassemblés sur le tableau ci-contre.

Ces différences portent sur l'abondance du sédiment et sur la proportion des grains de pollens appartenant à la plante sur laquelle le miel a été 
récolté. Mais elles sont encore plus nettes si on compare les nombres de grains de pollens provenant de cette plante. Ainsi un miel de Lavandin en section donne à la centrifugation un sédiment très réduit avec une richesse en pollen de Lavandin assez faible et un nombre de grains de cette plante très bas. En effet dans la fleur de Lavandin chaque étamine possède bien autant de grains de pollen que l'étamine de Lavandula vera, mais ce pollen ne contient pas de protoplasma, il est réduit à l'état d'un sac vide et son faible volume fait qu'il ne sort pas de l'étamine, ajoutons à cela que le I avandin est très nectarifère et qu'au moment de la meillée il n'existe pratiquement aucune plante pollinifère ce qui réduit considérablement les possibilités d'élevage pour la colonie. Ainsi se trouvent rassemblées plusieurs conditions favorables pour que ce miel soit très pauvre en pollen, et pour que la proportion en pollen de cette espèce soit extrêmement basse.

Analyses polliniques de miels unifloranx.

\begin{tabular}{|c|c|c|c|c|}
\hline \multirow[b]{2}{*}{ Miel de : } & & \multirow[b]{2}{*}{$\begin{array}{l}\text { Polleus } \\
\text { totaux }\end{array}$} & \multicolumn{2}{|c|}{$\begin{array}{c}\text { Pollens de la plante } \\
\text { mellifére }\end{array}$} \\
\hline & & & $\%$ & $\begin{array}{l}\text { Nombre } \\
\text { de grains } \\
\text { par ro r } \\
\text { de miel }\end{array}$ \\
\hline Lavandin & Section. & 750 & 2.3 & 172 \\
\hline Lavandin ........... & Extraction. & 3500 & 4 & 140 \\
\hline Lavandula rera D. C. & Extraction. & 2750 & $i^{1}$ & I 950 \\
\hline Satureia montana L. . & Section. & 6000 & 98 & 5880 \\
\hline Satureia montana L. . & Extraction. & 10600 & 56 & 5940 \\
\hline Rosmarinus officinalis L... & Extraction. & 11000 & 81 & 8910 \\
\hline Rosmarinus officinalis L... & Extraction. & 20000 & 72 & $I+400$ \\
\hline Calluna vulgaris $\mathrm{L} . . . .$. & Section. & $r+\infty 00$ & 99 & I. 860 \\
\hline Lippia canescens Н. В. K. & Extraction. & 22500 & 94 & 21150 \\
\hline Hedysarum coronarium L. . . & Extraction. & 22500 & 77 & I 7325 \\
\hline Paliurus Spina Christi Miller & Extraction. & 25000 & 75 & I8 750 \\
\hline Myosotis alpestris Schm...... & Extraction. & Iof 500 & 82 & $8733^{\circ}$ \\
\hline Castanea sativa Miller $\ldots \ldots \ldots \ldots \ldots \ldots$ & Extraction. & 135000 & 95 & I $2825^{\circ}$ \\
\hline
\end{tabular}

En ce qui concerne les miels de Lavandula et de Satureia, on doit considérer que ces plantes fleurissent à une époque de l'année où l'élevage est réduit par suite de la sécheresse, et que la flore d'accompagnement est très maigre. Il en résulte une proportion élevée de pollen de ces espèces mais une faible valeur du nombre de pollens de ces plantes mellifères.

Le cas du Rosmarinus est différent, cette plante fleurit au printemps, les possibilités d'élevage sont nettement plus favorables et malgré la position des étamines qui sont très éloignées du tube de la corolle, le miel récolté par les abeilles est sensiblement plus riche en pollen.

Pour Lippia, Hedysarum et Paliurus la disposition des étamines n'est pas défavorable et le nombre de pollens de ces plantes est nettement plus élevé.

En ce qui concerne $M$ yosotis et Castanea ; les miels sont très riches en 
pollens de ces espèces. 'Toutefois pour le miel de Castanea le nombre des pollens totaux est relativement faible, mais il semble qu'il existe pour cette dernière espèce du miel de fleur et du miellat, ce dernier serait beancoup plus riche en pollen.

\section{Conclusions.}

L.es quelques miels unifloraux étudiés permettent de constater les grandes différences existant entre le nombre de grains de pollen apportés par une plante mellifère à son propre miel. Suivant les espèces envisagées ce nombre varie de I72 à I 28250 pollens. On peut remarquer également que si le spectre pollinique des miels de I,avandin et de Satureia varie beaucoup, selon le mode d'extraction le nombre de grains ne varie pratiquement pas. Par contre il semble que ce mode d'extraction a une action si la plante fleurit en période d'élevage : cas du miel de Rosmarinus dont les 2 analyses sont bien différentes.

Par ailleurs ces résultats en faisant connaître des miels très pauvres en pollens ne manqueront pas d'attirer l'attention du palynologiste sur ces miels dont la richesse en pollen fait penser à des sirops de nourrissement, ou à ces miels récoltés par l'apiculteur en fin de saison et dont l'interprétation des résultats de l'analyse pollinique apparait très ardue par suite du mélange de miels ayant des richesses très différentes.

Nous pensons que le jour où tous les miels unifloraux seront connus et que leurs caractéristiques polliniques ainsi que physiques (couleur, parfum) et chimiques seront définies, un grand pas sera fait pour faciliter le travail des spécialistes, qui par suite des échanges commerciaux peuvent avoir à examiner des miels inconnus pour eux. 